\title{
Application of Variational Mode Decomposition and Permutation Entropy for Rolling Bearing Fault Diagnosis
}

\author{
Xiaoxia Zheng, Guowang Zhou and Dongdong Li \\ Shanghai University of Electric Power, Shanghai, China. \\ Rongcheng Zhou and Haohan Ren \\ Shanghai Donghai Wind Power Co., Ltd, Shanghai, China.
}

(Received 6 December 2016; accepted 21 March 2018)

\begin{abstract}
Rolling bearings are the key components of rotating machinery. However, the incipient fault characteristics of a rolling bearing vibration signal are weak and difficult to extract. To solve this problem, this paper presents a novel rolling bearing vibration signal fault feature extraction and fault pattern recognition method based on variational mode decomposition (VMD), permutation entropy (PE) and support vector machines (SVM). In the proposed method, the bearing vibration signal is decomposed by VMD, and the intrinsic mode functions (IMFs) are obtained in different scales. Then, the PE values of each IMF are calculated to uncover the multi-scale intrinsic characteristics of the vibration signal. Finally, PE values of IMFs are fed into SVM to automatically accomplish the bearing condition identifications. The proposed method is evaluated by rolling bearing vibration signals. The results indicate that the proposed method is superior and can diagnose rolling bearing faults accurately.
\end{abstract}

\section{INTRODUCTION}

In order to ensure the reliability of rotating machinery, condition monitoring and fault diagnosis of rolling bearings timely and accurately become very essential. ${ }^{1}$ Because of the direct connection between the structure of the rotating machinery and vibration sensor, vibration analysis methods have been widely applied for fault diagnosis in machinery fields. ${ }^{2,3}$ When a fault occurs on the surface of a rolling bearing, the periodicity impulses will appear in the vibration signals. Vibration signals are directly associated with the structural dynamics of the monitored machine, which consists of adequate information relating to the mechanical system. How to extract useful information that represents the bearing fault feature from the vibration signals is the key point for condition monitoring and fault diagnosis. ${ }^{4}$ Due to the complex and harsh working conditions of the mechanical system, the useful characteristics of vibration signals are usually drowned out by noises. ${ }^{5}$ Many advance signal processing methods have been employed to extract the characteristics of the vibration signals.

The well-known time-frequency method wavelet transform (WT) can decompose a signal into several scale timefrequency components. The WT method is widely used in the fault diagnosis of rolling bearings. ${ }^{6,7}$ However, the appropriate selection of the wavelet basis function needs to be solved, and WT does not have the self-adaptive feature. Empirical mode decomposition method $(\mathrm{EMD})^{8}$ is a self-adaptive signal decomposition technique, which can decompose any complicated signal into IMFs depending on the natural oscillations embedded in signal. The EMD method has received the extensive attention of researchers and has been used in the diagnosis appli- cations of mechanical system. ${ }^{9}$ However, the mode mixing and end effect problems of EMD algorithm limit its applications to the fault diagnosis. ${ }^{10,11}$ Ensemble empirical mode decomposition (EEMD), ${ }^{12}$ an improved version of EMD, improves the mode mixing drawback of the EMD method. The EEMD method consists of sifting through an ensemble of white noiseadded signals and treats the mean as the final true result. The EEMD method has improved the mode mixing, and has been applied to bearing fault diagnosis. ${ }^{13}$ Xiang and Zhong, ${ }^{14}$ on the basis of EEMD, found that feature extraction is further performed by the random decrement technique (RDT) to determine the feature frequency, and the combination of the methods is effective for fault diagnosis. Considering that the faulty component has severe noise, LI et al. proposed the morphological filtering-translation invariant wavelet and the improved EEMD method, which reduced the noise of the original signal and made the diagnosis more accurate..$^{15}$

Recently, a new adaptive and quasi-orthogonal signal decomposition method, variational mode decomposition (VMD) was put forward by Konstantin Dragomiretskiy and Dominique Zosso. ${ }^{16}$ VMD can decompose a signal into various modes or IMFs by using calculus of variation. VMD is a signal decomposition algorithm with more refinement time-frequency divisions than EMD and local mean decomposition (LMD). ${ }^{17}$ The decomposition performance of VMD can substitute EMD method which does not have a strong mathematical foundation. ${ }^{18}$ Considering the advantage in the aspects of signal decomposition, VMD is applicable to process the rolling bearing vibration signals.

In order to extract the dynamic characteristics hidden in the 
measured signals, many nonlinear parameter estimation techniques emerge in fault diagnosis domain. Yang et al. ${ }^{19}$ used fractal dimension to classify rolling element bearing fault types and evaluate various fault conditions. Yan and Gao introduced approximate entropy (ApEn) to characterize the severity of structural defect. ${ }^{20}$ The fuzzy entropy method is used to extract fault characteristics of the rolling bearing vibration signals. ${ }^{21}$ The fractal demands very long data set, which is not suitable for online monitoring. Although the methods based on entropy require much less computation and are simplicity, they have good properties in fault diagnosis of bearing. ${ }^{22}$

Permutation entropy (PE) was presented by Bandit and Pompe. PE can estimate the complexity of time series through the comparison of neighboring values. ${ }^{23}$ Permutation entropy is robust under the non-liner distortion of signals and has a fast calculation speed. The PE method has been widely applied to detect dynamic changes of complex time series, such as electroencephalograph (EEG) signals ${ }^{24}$ and cardiac heart inter-beat signals. ${ }^{25}$ In the mechanical fault diagnosis field, many researchers investigated the PE method and the studies show that PE can effectively detect and amplify the dynamic changes of the vibration signal. Yan et al. used PE as an effective feature to monitor the working conditions of rolling bearings. ${ }^{26}$ Zhang et al. extracted the characteristics of the vibration signal by calculating the PE value of the IMFs after processing the vibration signal by EEMD, and then utilized a support vector machine (SVM) to recognize bearing faults. ${ }^{27}$ The effectiveness of permutation entropy is also been proved in gearbox fault diagnosis. ${ }^{28}$

The purpose of this study is to propose a novel method based on VMD, PE and SVM for rolling bearing fault feature extraction and identification. Considering the advantages of VMD in signal processing, PE value of IMFs are calculated as feature vectors that representing the multi-scale characteristics of the vibration signal. After extracting features using the VMD and $\mathrm{PE}$ algorithm, the obtained features are fed into one against all (OAA) $\mathrm{SVM}^{29}$ classifier to accomplish the fault recognition. The vibration signals are sampled from rolling bearing. Then the signals are decomposed into several IMFs by VMD. Subsequently, PE values of IMFs would be calculated and utilized as the feature vectors. Finally, the feature vectors of the vibration signals are fed in the SVM model to identify the bearing conditions. The rest of this paper is organized as follows. In Section 2, the VMD algorithm and its number of modes are studied. PE and its parameter settings are both described and explored in Section 3. The procedures of the proposed method will be presented in Section 4. The experimental results of the proposed method will be discussed in Section 5. Finally, conclusions are drawn in Section 6.

\section{VARIATIONAL MODE DECOMPOSITION}

\subsection{Variational Mode Decomposition Algorithm}

Variational mode decomposition is a newly adaptive decomposition method, which can decompose the signal into various modes or band-limited intrinsic mode functions by using calculus of variation. VMD can decompose an input signal $f$ into a discrete number of sub-signals $u_{k}$, that have specific sparsity properties of its bandwidth in the spectral domain. Each mode $k$ is mostly compact around a center pulsation $\omega_{k}$, which is to be determined along with the decomposition.

Intrinsic mode function is amplitude modulated - frequency modulated (AM-FM) signal, written as:

$$
u_{k}(t)=A_{k}(t) \cos \left(\phi_{k}(t)\right)
$$

where the phase $\phi_{k}(t)$ is a non-decreasing function, $\phi_{k}^{\prime}(t) \geq 0$, the envelope is non-negative $A_{k}(t) \geq 0$, both the envelope $A_{k}(t)$ and the instantaneous frequency $\omega_{k}(t)=\phi_{k}^{\prime}(t)$ vary much slower than the phase $\phi_{k}(t){ }^{16}$

In order to measure the bandwidth of an IMF $u_{k}(t)$, Dragomiretskiy and Zosso proposed a function in their study. For each mode $u_{k}(t)$, using the Hilbert transform to obtain a unilateral frequency spectrum of the associated analytic signal:

$$
\left(\delta(t)+\frac{j}{\pi t}\right) * u_{k}(t)
$$

Frequency spectrum of the analytic signal assumed to be centered on $\omega_{k}$. By multiplying the analytic signal with $e^{-j \omega_{k} t}$, the signal frequency is translated to be centered at origin:

$$
\left[\left(\delta(t)+\frac{j}{\pi t}\right) * u_{k}(t)\right] e^{-j \omega_{k} t} .
$$

Using $H^{1}$ Gaussian smoothness of the demodulated signal the bandwidth of each mode is estimated. The resulting constrained variational problem is the following:

$$
\begin{array}{r}
\min _{\left\{u_{k}\right\},\left\{\omega_{k}\right\}}\left\{\sum_{k}\left\|\partial_{t}\left[\left(\delta(t)+\frac{j}{\pi t}\right) * u_{k}(t)\right] e^{-j \omega_{k} t}\right\|_{2}^{2}\right\} \\
\text { s.t. } \sum_{k=1}^{K} u_{k}=f ;
\end{array}
$$

where $\left\{u_{k}\right\}=\left\{u_{1}, u_{2}, \ldots, u_{K}\right\}$ and $\left\{\omega_{k}\right\}=\left\{\omega_{1}, \omega_{2}, \ldots, \omega_{K}\right\}$ are the set of all modes and their center frequencies, $f$ is the original signal. The calculations will be easier by converting constrained problem to unconstrained problem. The augmented Lagrangian multiplier method and a quadratic penalty is used to convert the problem into a unconstrained optimization problem as follows:

$$
\begin{array}{r}
L\left(\left\{u_{k}\right\},\left\{\omega_{k}\right\}, \lambda\right)= \\
=\alpha \sum_{k=1}^{K}\left\|\partial_{t}\left[\left(\delta(t)+\frac{j}{\pi t}\right) * u_{k}(t)\right] e^{-j \omega_{k} t}\right\|_{2}^{2}+ \\
+\left\|f(t)-\sum_{k=1}^{K} u_{k}(t)\right\|_{2}^{2}+\left\langle\lambda(t), f(t)-\sum_{k=1}^{K} u_{k}(t)\right\rangle ;
\end{array}
$$

where $\lambda$ is Lagrangian multiplier, $\alpha$ is the balancing parameter of the data-fidelity constraint.

The alternate direction method of multipliers (ADMM) ${ }^{30}$ is used to solve the original minimization problem. In ADMM 
algorithm, the solutions of one variable is solved assuming the other variables are known. The expression for updating $u_{k}$ at the $n+1$ ' iteration is written as:

$$
\begin{array}{r}
u_{k}^{n+1}= \\
\underset{u_{k} \in X}{\arg \min }\left\{\alpha\left\|\partial_{t}\left[\left(\delta(t)+\frac{j}{\pi t}\right) * u_{k}(t)\right] e^{-j \omega_{k} t}\right\|_{2}^{2}+\right. \\
\left.+\left\|f(t)-\sum_{i} u_{i}(t)+\frac{\lambda(t)}{2}\right\|_{2}^{2}\right\} ;
\end{array}
$$

where $\omega_{k}$ denotes the $\omega_{k}^{n+1}, \sum_{i} u_{i}(t)$ denotes $\sum_{i \neq k} u_{i}(t)^{n+1}$.

Making use of the Parseval/Plancherel Fourier isometry under the $L^{2}$ norm, the problem of the formulas solved in spectral domain:

$$
\begin{array}{r}
\hat{u}_{k}^{n+1}= \\
=\underset{\hat{u}_{k}, u_{k} \in X}{\arg \min }\left\{\alpha\left\|j \omega\left[\left(1+\operatorname{sgn}\left(\omega+\omega_{k}\right)\right) \hat{u}_{k}\left(\omega+\omega_{k}\right)\right]\right\|_{2}^{2}+\right. \\
\left.+\left\|\hat{f}(\omega)-\sum_{i} \hat{u}_{i}(\omega)+\frac{\hat{\lambda}(\omega)}{2}\right\|_{2}^{2}\right\} .
\end{array}
$$

The variable $\omega$ can be replaced by $\omega-\omega_{k}$ in the first term:

$$
\begin{array}{r}
\hat{u}_{k}^{n+1}= \\
=\underset{\hat{u}_{k}, u_{k} \in X}{\arg \min }\left\{\alpha\left\|j\left(\omega-\omega_{k}\right)\left[(1+\operatorname{sgn}(\omega)) \hat{u}_{k}(\omega)\right]\right\|_{2}^{2}+\right. \\
\left.+\left\|\hat{f}(\omega)-\sum_{i} \hat{u}_{i}(\omega)+\frac{\hat{\lambda}(\omega)}{2}\right\|_{2}^{2}\right\} .
\end{array}
$$

In the above expression, the first term vanishes for negative frequencies:

$$
\begin{array}{r}
\hat{u}_{k}^{n+1}= \\
=\underset{\hat{u}_{k}, u_{k} \in X}{\arg \min }\left\{\int_{0}^{\infty} 4 \alpha\left(\omega-\omega_{k}\right)^{2}\left|\hat{u}_{k}(\omega)\right|^{2}+\right. \\
\left.+2\left|\hat{f}(\omega)-\sum_{i} \hat{u}_{i}(\omega)+\frac{\hat{\lambda}(\omega)}{2}\right|^{2} \partial \omega\right\} .
\end{array}
$$

The solution of this quadratic optimization problem is readily found by letting the first variation vanish for the positive frequencies:

$$
=\left(\hat{f}(\omega)-\sum_{i \neq k} \hat{u}_{i}(\omega)+\frac{\hat{\lambda}(\omega)}{2}\right) \frac{\hat{u}_{k}^{n+1}(\omega)=}{1+2 \alpha\left(\omega-\omega_{k}\right)^{2}} ;
$$

where $\omega_{k}$ is computed at the center of gravity of the corresponding mode's power spectrum. It can be seen that Wiener filtering is embedded in the VMD algorithm, which makes it much more robust to sampling and noise. In order to obtain the mode in time domain, inverse Fourier transform is applied to the filtered analytic signal.

To update frequencies $\omega_{k}$, the relevant problem is as following:

$$
\begin{array}{r}
\omega_{k}^{n+1}= \\
=\underset{\omega_{k}}{\arg \min }\left\{\left\|\partial_{t}\left[\left(\delta(t)+\frac{j}{\pi t}\right) * u_{k}(t)\right] e^{-j \omega_{k} t}\right\|_{2}^{2}\right\} .
\end{array}
$$

As before, the optimization can be transferred to spectral domain:

$$
\begin{array}{r}
\omega_{k}^{n+1}= \\
=\underset{\omega_{k}}{\arg \min }\left\{\int_{0}^{\infty}\left(\omega-\omega_{k}\right)^{2}\left|\hat{u}_{k}(\omega)\right|^{2} \partial \omega\right\} .
\end{array}
$$

The quadratic problem is solved as:

$$
\omega_{k}^{n+1}=\frac{\int_{0}^{\infty} \omega\left|\hat{u}_{k}(\omega)\right|^{2} \partial \omega}{\int_{0}^{\infty}\left|\hat{u}_{k}(\omega)\right|^{2} \partial \omega} .
$$

Update for $\lambda$ :

$$
\lambda^{n+1}=\lambda^{n}+\tau_{a}\left(f-u_{k}^{n+1}(t)\right) ;
$$

where $\tau_{a}$ is time-step of the dual ascent.

Variational mode decomposition complete algorithm is as follows:

Initialize $\left\{\hat{u}_{k}^{1}\right\},\left\{\hat{\omega}_{k}^{1}\right\}, \hat{\lambda}^{1}, n \leftarrow 0$

\section{Repeat}

$$
\begin{aligned}
& n \leftarrow n+1 \\
& \text { or } k=1: K \text { do }
\end{aligned}
$$

Update $\hat{u}_{k}$ for all $\omega \geq 0$ :

$$
\hat{u}_{k}^{n+1}(\omega) \leftarrow \frac{\hat{f}(\omega)-\sum_{i<k} \hat{u}_{i}^{n+1}(\omega)-\sum_{i>k} \hat{u}_{i}^{n}(\omega)+\frac{\hat{\lambda}^{n}(\omega)}{2}}{1+2 \alpha\left(\omega-\omega_{k}^{n}\right)^{2}} .
$$

Update $\omega_{k}$ :

$$
\omega_{k}^{n+1} \leftarrow \frac{\int_{0}^{\infty} \omega\left|\hat{u}_{k}^{n+1}(\omega)\right|^{2} \partial \omega}{\int_{0}^{\infty}\left|\hat{u}_{k}^{n+1}(\omega)\right|^{2} \partial \omega}
$$

end for

Dual ascent for all $\omega \geq 0$ :

$$
\hat{\lambda}^{n+1}(\omega) \leftarrow \hat{\lambda}^{n}(\omega)+\tau_{a}\left(\hat{f}(\omega)-\sum_{k} \hat{u}_{k}^{n+1}(\omega)\right) .
$$

Until convergence: $\sum_{k}\left\|\hat{u}_{k}^{n+1}-\hat{u}_{k}^{n}\right\|_{2}^{2} /\left\|\hat{u}_{k}^{n}\right\|_{2}^{2}<\varepsilon$

\subsection{Setting the Number of Modes}

VMD algorithm and EMD algorithm are based on two different theoretical frameworks. Unlike EMD, the signal processing by VMD requires a predetermined number of modes. The different number of modes of decomposition will have an impact on the decomposition results and then the final fault diagnosis. Selecting the appropriate number of modes of decomposition is crucial to the proposed fault diagnosis method. This research determines the number of modes $K$ by analyzing and comparing the spectrum of original signal and decomposed IMFs, and then proposes the general principles of determination number of modes.

The vibration data of roller bearings in this paper come from Case Western Reserve University (CWRU). ${ }^{31}$ The outrace fault bearing vibration signal is decomposed into different sets of modes, or different $K$ values of VMD algorithms. 


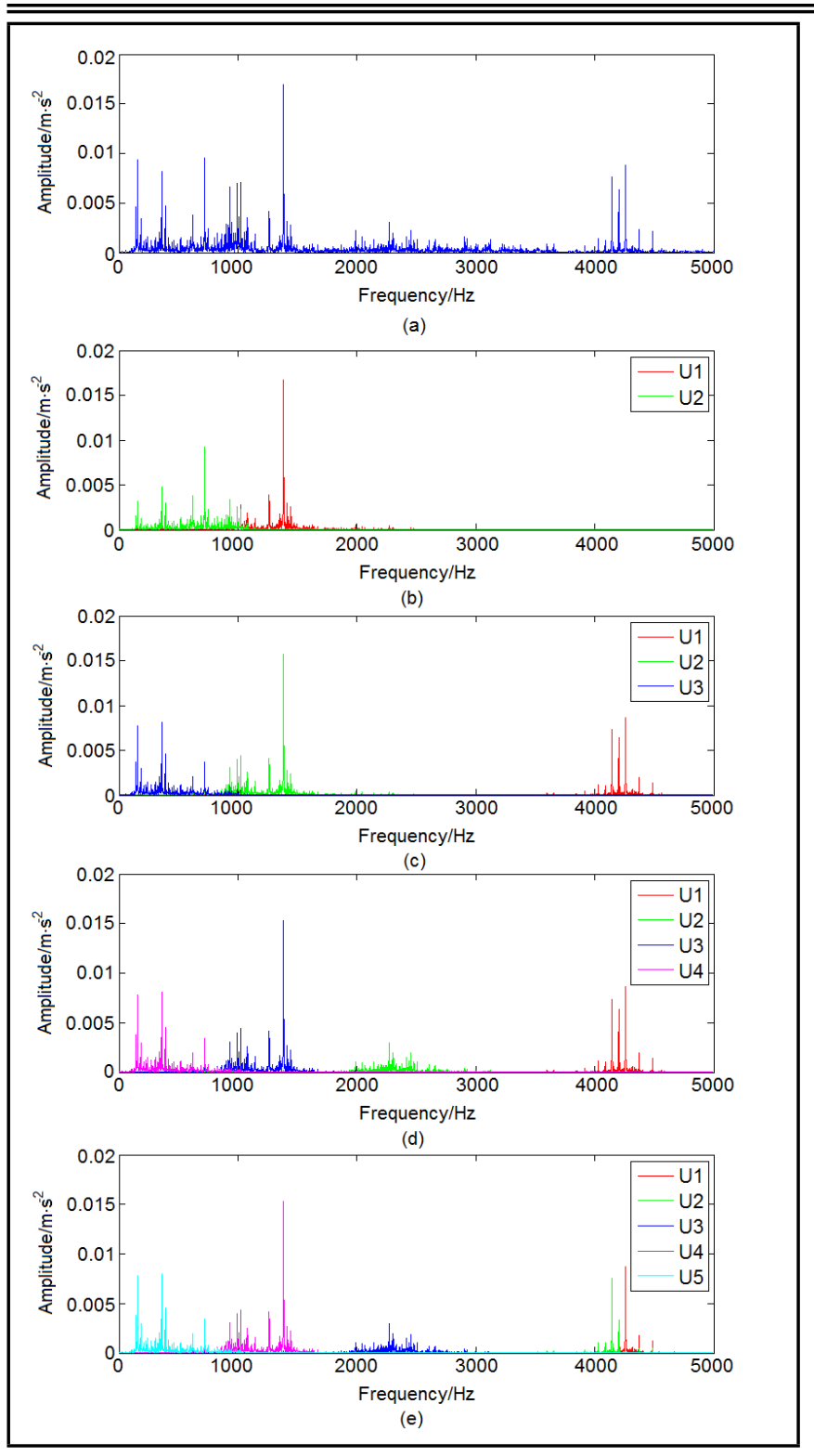

Figure 1. Spectrum of original signal and each $K$ mode components: (a) original signal, (b) $K=2$, (c) $K=3$, (d) $K=4$, (e) $K=5$.

To avoid the influence of other factors, balancing parameters were set as constants. Figure 1 shows the spectrum of each set mode components and the original vibration signal spectrum. The spectrum shows that different sets of modes had effects on the result of the signal decomposition. When $K=2$ (too few modes), the VMD algorithm is equivalent to the adaptive Wiener filter, the important information will be discarded. Indeed, as shown in Fig. 1b, the high frequency band above $2000 \mathrm{~Hz}$ is mostly discarded. If the characteristic information is contained in the high frequency band, the important information will be missing after VMD algorithm processing in this condition.

On the other hand, when $K=5$ (too many modes), the same parts of the signal spectrum are shared by two or more different modes. As shown in Fig. 1e, some frequency of mode $\mathrm{U} 1$ and mode $\mathrm{U} 2$ are overlap, their center frequencies coincide. The characteristic information will be shared by two or more modes, this result in the weak signal fault characteristic like the incipient fault of rolling bearing is difficult to be identified.

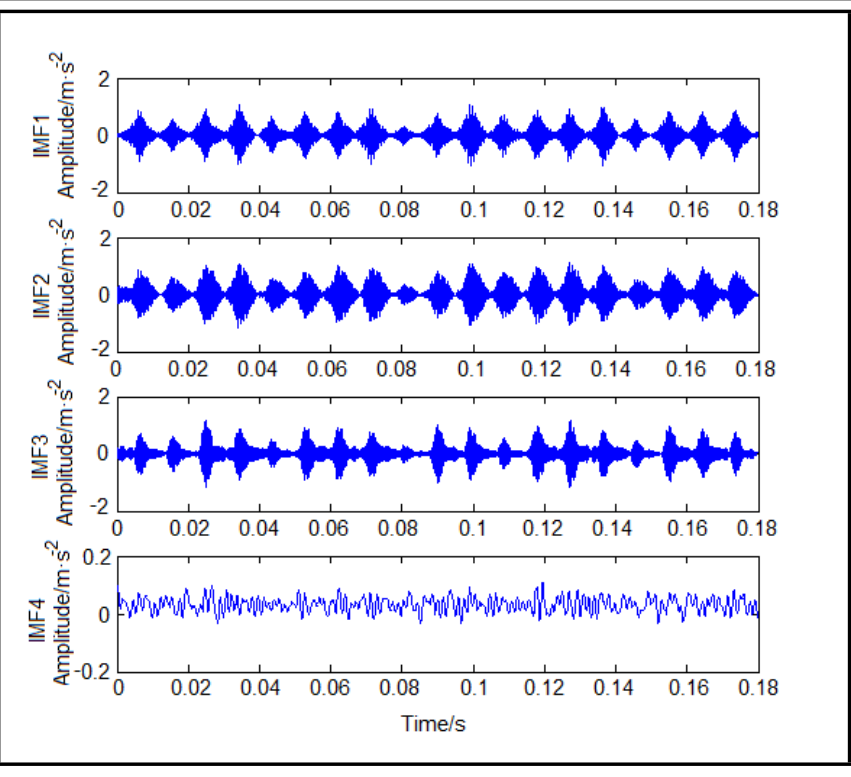

Figure 2. VMD results of bearing vibration signal with outer race fault.

Based on the above analysis, the general principles of determine the number of modes decomposition when extracting signal characteristic using the VMD algorithm is proposed as follows:

(1) Avoid mode mixing. Mode mixing will reduce the intensity of characteristic information, which increase the difficulty of weak information extraction. Many researchers have studied the mode mixing problem of the EMD algorithm and proposed the improved methods. ${ }^{12,32}$ But, as long as the appropriate number of $K$ modes are being chosen, the mode mixing problem of the VMD algorithm can be avoided.

(2) Reserve all characteristics. The main purpose of decomposing signal is to extract the information that the original signal contains. We hope that the modes decomposition contains all the feature of the decomposed signal, which can extract the useful information instead of discarding the useful information.

According to the above principles and comparing the different number of modes and the original signal spectrum in Fig. 1 shows: both $K=2$ (too few modes) and $K=5$ (too many modes) is not the appropriate sets of the decomposition and the frequency band $2000 \mathrm{~Hz}-4000 \mathrm{~Hz}$ are mostly discarded when $K=2$. So, $K=4$ is a more appropriate set for the decomposition when analyzing this signal. The results processed by VMD are shown in Fig. 2.

\section{PERMUTATION ENTROPY}

\subsection{Basic Principle of Permutation Entropy}

The concept of entropy stems from the ideas of physics, which measures the disorder degree of a system. The larger entropy value indicates a more complex system. Permutation entropy (PE) was proposed by Bandt and Pompe ${ }^{23}$ for detecting the dynamic change of the time series. According to the Takens-Maine theorem, the phase space of a time series 
$\{X(i), i=1,2, \ldots, N\}$ can be reconstructed as:

$$
\left[\begin{array}{cccc}
x(1) & x(1+\tau) & \ldots & x(1+(m-1) \tau) \\
\vdots & \vdots & & \vdots \\
x(i) & x(i+\tau) & \ldots & x(i+(m-1) \tau) \\
\vdots & \vdots & & \vdots \\
x(G) & x(G+\tau) & \ldots & x(G+(m-1) \tau)
\end{array}\right]
$$

where $m$ is the embedded dimension, it is an important factor in determining the reconstruction quality of phase space. The proper value of $m$ can correctly estimate the topological characteristics of the original state space. $\tau$ is the time delay value. The theory holds that any delay time value can be taken in a noise-free, infinitely long one-dimensional time series. However, in practice, general time series contain noise and have limited length. Therefore, it is necessary to select an appropriate delay time value and extract the feature vectors of the phase space. $G$ is the number of vectors in phase space reconstruction, $G=N-(m-1) \tau$. $m$ number of real values contained in each $x(i)$ can be arranged in an increasing order as:

$$
\begin{array}{r}
\left\{x\left(i+\left(j_{1}-1\right) \tau\right) \leq x\left(i+\left(j_{2}-1\right) \tau\right) \leq\right. \\
\left.\leq \ldots \leq x\left(i+\left(j_{m}-1\right) \tau\right)\right\}
\end{array}
$$

where $j_{1}, j_{2}, \ldots, j_{m}$ is the column index of the reconstruction component elements.

For a given embedding dimension, it will be $m$ ! possible permutations. If each permutation is considered as a symbol sequence, $l$, the probability distribution is $P_{l}$. Thus, based on the Shannon entropy definition the permutation entropy of a given time series $\{X(i), i=1,2, \ldots, N\}$ can be defined as:

$$
P E(m)=-\sum_{l=1}^{k} P_{l} \ln P_{l} .
$$

The permutation entropy of order $m$ can be normalized as:

$$
P E=P E(m) / \ln (m !) ;
$$

where $1 / \ln (m !)$ is a normalization factor such that $0 \leq$ $P E(m) / \ln (m !) \leq 1$. The value of permutation entropy can represent the randomicity of the time series, and it describes local order structure of the time series. It can be known that a bigger PE value represents more random and irregular of time series. The smaller the value of PE, the more regular the time series is.

\subsection{Parameters Setting for Permutation Entropy}

In the calculation of permutation entropy, it is necessary to consider and determine the parameters, such as data length $N$, embedded dimension $m$ and time delay. Different parameters will have impact on the calculation of the permutation entropy. In order to investigate the effect of parameters on PE calculation, we calculate the PE values of rolling bearing vibration signal as the time series under different value of parameters.

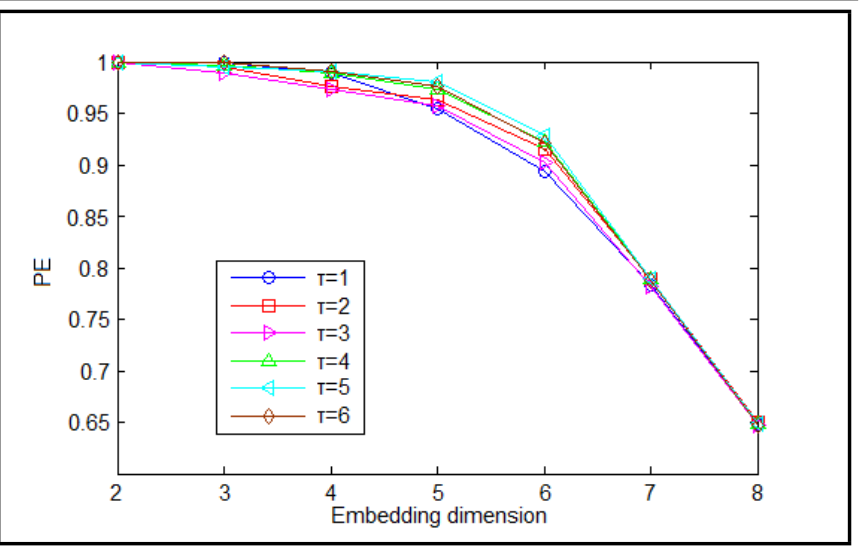

Figure 3. The PE values of vibration signals with different time delays.

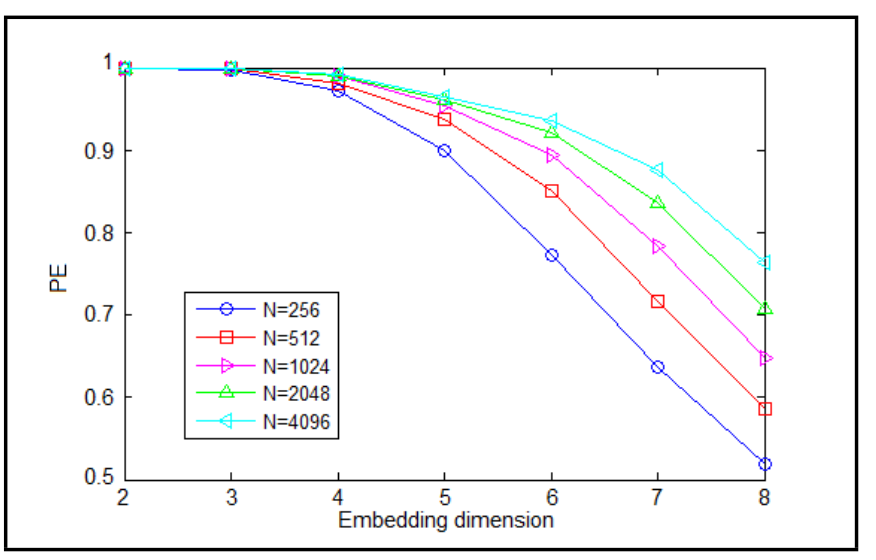

Figure 4. The PE values of vibration signals with different lengths.

The relationship between PE value and time delay is shown in Fig. 3, where PE calculated by selecting the data length $N=$ 1024 , time delay $\tau=1-6$, under the dimension $m=2-8$. We can clearly see that when $\tau$ takes $1-6$, there are no significant changes with the same embedded dimension. Thus, time delay $\tau=1$ is used to calculate the PE in this paper.

Bandt and Pompe ${ }^{23}$ recommended the value of embedded dimension $m=3-7$. If the embedded dimension is too small, such as 1 or 2, permutation entropy cannot detect the dynamic changes of the mechanical vibration signals exactly. On the other hand, when $m$ is too large, reconstruction of phase space will homogenize vibration signals, and it will be difficult to detect changes in time series. ${ }^{27}$ Cao and Tung ${ }^{33}$ found that the value $m=5,6$ or 7 seems to be the most suitable for capturing the periodic windows in a transient time series. After a series of test, we set $m=6$ in this paper.

The data length is also an important parameter in the calculation of the PE value. In the process of calculation, it is found that, too small $N$ would cause computational results to be no statistical significance. However, in order to accurately find transitional signals, data length $N$ should not be too large either. Figure 4 shows PE values of vibration signals with data length $N=256,512,1024,2048$ and 4096 by selecting $m=$ $2-8, \tau=1$. It can be seen that when embedded dimension $m \leq 5$, PE values are small change with the increase of the data length. Table 1 shows the permutation entropy and the differences between the different data length of the vibration signal. We can see that the permutation entropy difference de- 
Table 1. PE and PE differences of vibration signal with different lengths.

\begin{tabular}{||c|c|c|c|c|c|}
\hline \hline Data length & 256 & 512 & 1024 & 2048 & 4096 \\
\hline PE & 0.772 & 0.85 & 0.894 & 0.922 & 0.935 \\
\hline Difference & 0.078 & 0.044 & 0.028 & 0.013 & - \\
\hline
\end{tabular}

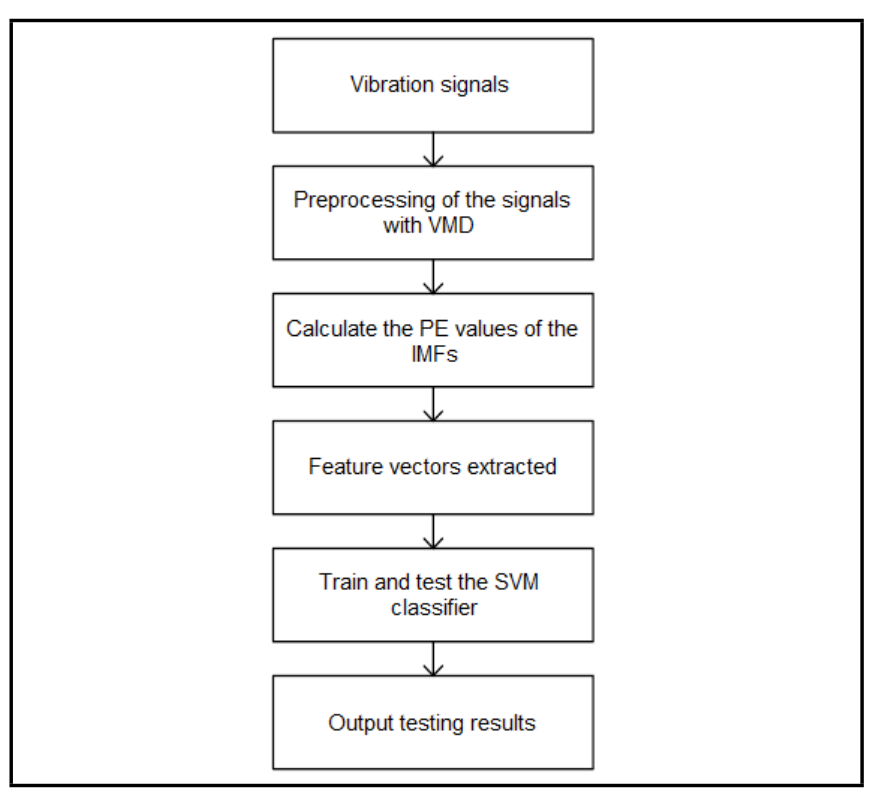

Figure 5. Flow chart of the proposed method.

creases with the increase of the data length. PE value is stabilized when the data length is larger than 1024. Thus, we set data length $N=2048$ to calculate the permutation entropy of the rolling bearing vibration signal in this paper.

\section{THE PROPOSED METHOD}

We proposed a hybrid approach using SVM and PE to extract the characteristic information, and SVM to classify the fault types. The proposed method combing the advantages of VMD on signal decomposition and the property of the PE which can detect the randomness and dynamic mutation of vibration signals. The vibration signals of the rolling bearing are decomposed into sets of IMFs by variational mode decomposition. Then, the PE values of the IMFs are calculated to form the feature vectors. Finally, the fault feature vectors are fed into SVM to classify the faulty types. The complete process of the proposed method flow chart is shown in Fig. 5.

The main steps of the proposed method are as follows:

(1) Collect the normal status, inner race fault, outer race fault and ball fault vibration signals of rolling element bearing.

(2) Each vibration signal data is divided into several subsequences of a certain length.

(3) The vibration signal is processed by VMD and a set of IMF components will be obtained.

(4) PE values of IMF components are calculated, and the $P E$ values are considered as the fault feature vectors of subsequences signal.

(5) The feature vectors of different conditions are used to train the SVM, and the SVM classification models of each condition are obtained.

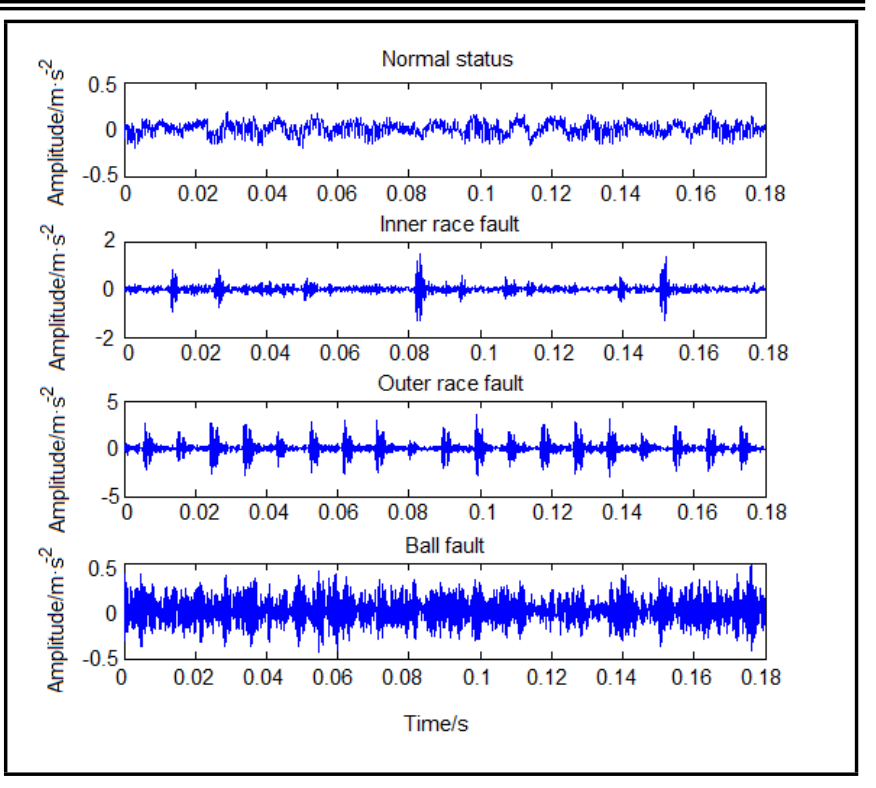

Figure 6. Vibration signals of rolling bearing under different working conditions.

(6) Test vibration signals are collected from the rolling bearing for diagnosis, and the feature vectors are obtained according to above steps. The feature vectors are put into the trained SVM to specify rolling bearing fault type.

\section{EXPERIMENTAL DATA ANALYSIS}

The experimental vibration data of roller bearings also come from Case Western Reserve University. The normal status, inner race fault, outer race fault and ball fault of the bearing vibration signals are divided into some non-overlapping sections with the data length $N=2048$. The four working conditions time waveform of rolling bearing vibration signals are shown in Fig. 6. Each condition has 40 samples, and there are total 160 samples. $25 \%$ samples of the total samples, namely 10 samples in each condition will be randomly selected as the training data, and the remaining samples are used as the testing data.

We applied the VMD algorithm to decompose the training vibration signals with four conditions. Four IMF components are obtained in each sample data. We then calculated the PE value of the IMF components to extract the fault features, and the average of PE feature vectors are shown in Fig. 7. A total 40 feature vectors collected from the training vibration signal of the four conditions were used to train the VMD classifier. In this study, a one against all SVM model of multi-class classification is used to distinguish the four bearing conditions. Permutation entropy feature of each condition is taken as a positive class, and the remaining three conditions as the negative class. Then input the SVM classifier to train the SVM prediction model.

The testing features of the remaining 120 testing samples are obtained according to the proposed method and fed into the well trained SVM classifier. The results are listed in Table 2 . The overall classification accuracy rate of the proposed method is $97.5 \%$. We can see from the results table that one inner race fault is diagnosed as ball fault, and two outer race 


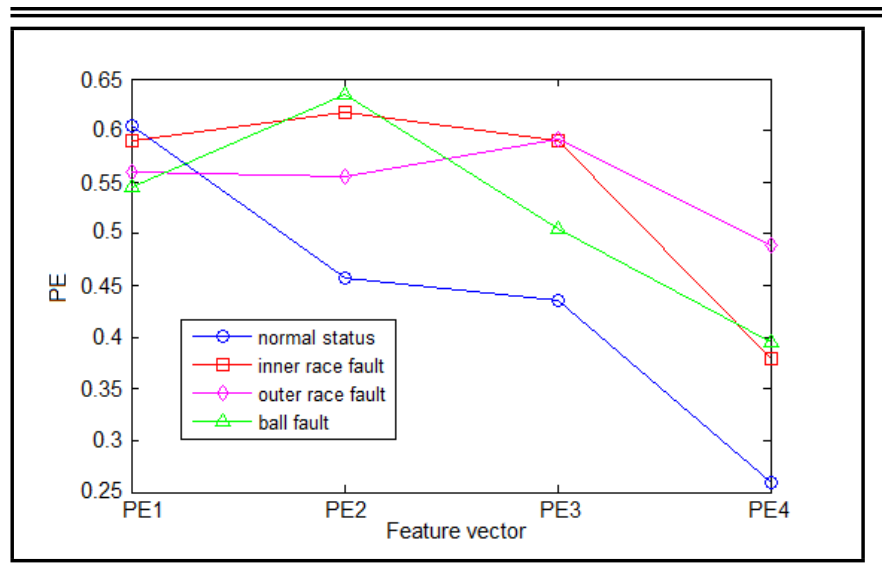

Figure 7. The feature vectors of PE.

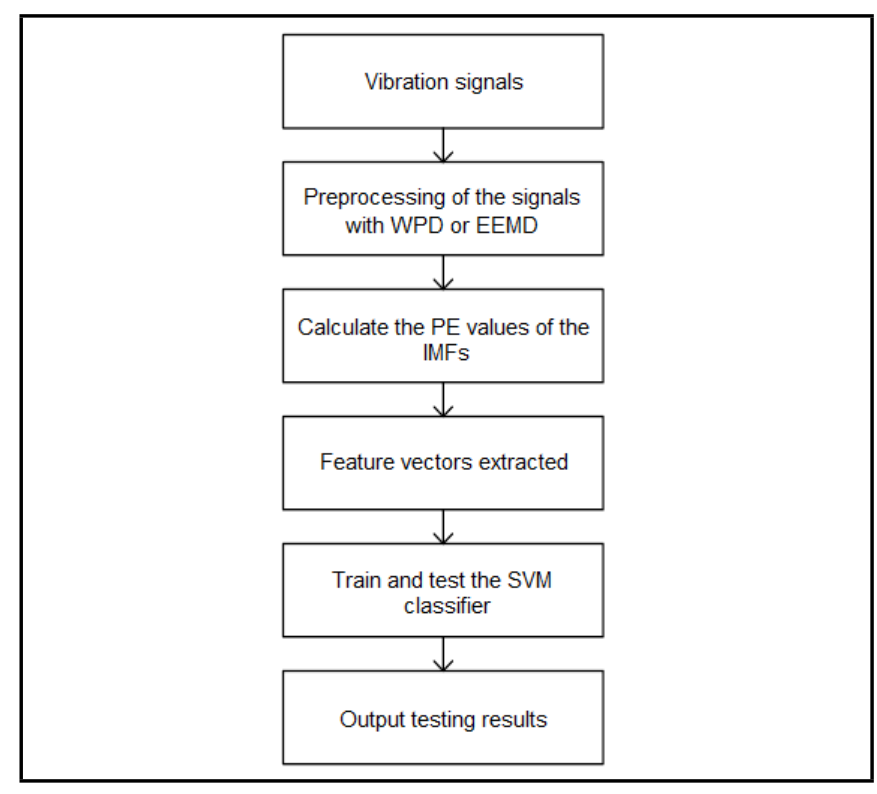

Figure 8. Flow chart of the comparison method.

faults are diagnosed as the inner race faults. While the accuracy of the normal status and the ball fault is $100 \%$.

In order to demonstrate the effects of the number of training samples, different training set sizes are selected to train SVM classification model, and the remaining samples are used to test the classification model. The results are presented in Table 3, where the percentages of training samples are $20 \%, 30 \%$, $40 \%, 50 \%$ and $60 \%$. The accuracy rate increases with the increase of the training samples. When the percentage of samples is $50 \%$, the proposed method accuracy is up to $100 \%$. Indeed, the large number of training samples will establish more accurate model. Whereas, it increases the computational complexity of the SVM algorithm.

To illustrate the important role of VMD decomposition, the proposed method is compared with those of wavelet packet decomposition (WPD) and EEMD decomposition, which use PE to extract the feature vectors and SVM as classifier. The process is shown in Fig. 8.

The selection and feature extraction method of vibration signals data of the bearing is same to the proposed method. We select the first four IMFs decomposed by EEMD, which almost contain all energy of the original signal, to extract the feature vectors. The wavelet packet scale is 2 , and the 4 wavelet coefficients are obtained to extract the feature vectors. The vectors were then used for training the SVM model and testing the classifier model. The classification results are listed in Table 4 and Table 5 .

In Table 4, normal status, inner race fault and ball fault recognition accuracy of EEMD method is $100 \%$. But 5 outer race fault samples are diagnosed as the inner race fault. Thus, the average accuracy of the EEMD method is $95.83 \%$, which is lower than the VMD method. Table 5 shows that the normal status and inner race fault recognition accuracy of the wavelet packet decomposition method is also $100 \%$. But 2 inner fault samples are diagnosed as ball fault, and 4 ball fault samples are diagnosed as inner race fault. The average accuracy of wavelet packet decomposition method is $95 \%$. It can be seen that the proposed feature extraction method can obtain a better success rate. From the comparison results we can see that the proposed method is efficient for rolling bearing fault diagnosis, and the overall classification rate is higher, to a certain extent, than ensemble empirical mode decomposition and wavelet packet decomposition.

\section{CONCLUSIONS}

Aiming at extracting characteristics information of rolling bearing vibration signal, a hybrid approach integrating VMD and $\mathrm{PE}$ is proposed in this work. The rolling bearing vibration signal is decomposed into a set of IMFs by VMD algorithm. PE values of IMFs are calculated as the feature of the vibration signal. The PE values are composed as feature vector and fed into SVM model to implement fault diagnosis. The normal, inner race fault, outer race fault and ball fault signals of rolling bearing are tested and verified.

The VMD algorithm is applied to rolling bearing vibration signals processing, which can decompose signal into bandlimited intrinsic mode functions and provide non-mode mixing and feature-rich data components for the following feature extraction and fault classification. Permutation entropy can detect the randomicity and dynamic mutations of vibration signals, and the characteristics information of rolling bearing vibration signals are extracted as much as possible combining with VMD. As compared to the EEMD and wavelet packet decomposition method, a higher classification rate is achieved by using the proposed. Therefore, the proposed method has a potential application for development of real time condition monitoring and fault diagnosis system.

\section{ACKNOWLEDGEMENTS}

This work supported by the National Natural Science Foundation of China (No.51507098), Green Energy Grid Engineering Technology of Shanghai Research Center (No.13DZ2251900), Shanghai Key Laboratory of Power Station Automation Technology (No.13DZ2273800) and Science Technology Commission of Shanghai Key Scientific Research Project (No.14DZ1200905). 


\begin{tabular}{l}
\hline \hline Table 2. Classification results based on VMD and PE. \\
\begin{tabular}{|c|c|c|c|c|c|c|c||}
\hline \multirow{2}{*}{ Fault type } & \multirow{2}{*}{ Test sample } & \multicolumn{7}{|c|}{ Classification results } & \multirow{2}{*}{ Classification rate (\%) } & $\begin{array}{c}\text { Overall } \\
\text { classification rate (\%) }\end{array}$ \\
\cline { 3 - 9 } & & Normal status & Inner race fault & Outer race fault & Ball fault & & \\
\hline Normal status & 30 & 30 & 0 & 0 & 0 & 100 & \\
\hline Inner race fault & 30 & 0 & 29 & 0 & 1 & 96.67 & \\
\hline Outer race fault & 30 & 0 & 2 & 28 & 0 & 93.33 & \\
\hline Ball fault & 30 & 0 & 0 & 0 & 30 & 100 & \\
\hline
\end{tabular}
\end{tabular}

Table 3. Classification results under different training samples.

\begin{tabular}{|c|c|c|c|c|c|}
\hline Training sample (\%) & 20 & 30 & 40 & 50 & 60 \\
\hline Classification rate (\%) & 96.1 & 98.21 & 98.89 & 100 & 100 \\
\hline
\end{tabular}

\section{REFERENCES}

1 Saidi L., Ali J. B., and Fnaiech F. Bi-spectrum based-EMD applied to the non-stationary vibration signals for bearing faults diagnosis. ISA transactions, 53 (5), 1650-1660, (2014). https://dx.doi.org/10.1016/j.isatra.2014.06.002

2 Janjarasjitt S., Ocak H., and Loparo K. A. Bearing condition diagnosis and prognosis using applied nonlinear dynamical analysis of machine vibration signal.Journal of Sound and Vibration, 317 (1), 112-126, (2008). https://dx.doi.org/10.1016/j.jsv.2008.02.051

3 Feng Z. P. and Zuo M. J. Vibration signal models for fault diagnosis of planetary gearboxes.Journal of Sound and Vibration, 331 (22), 4919-4939, (2012). https://dx.doi.org/10.1016/j.jsv.2012.05.039

4 Meng L. J. and Xiang J. W. Fault diagnosis of rolling bearing based on second generation wavelet denoising and morphological filter. Journal of Mechanical Science and Technology, 29 (8), 3121-3129, (2015). https://dx.doi.org/10.1007/s12206-015-0710-0

5 Peng Z. K., Peter W. T., and Chu F. L. A comparison study of improved Hilbert-Huang transform and wavelet transform: application to fault diagnosis for rolling bearing. Mechanical systems and signal processing, 19 (5), 974-988, (2005). https://dx.doi.org/10.1016/j.ymssp.2004.01.006

6 Rubini R. and Meneghetti U. Application of the envelope and wavelet transform analyses for the diagnosis of incipient faults in ball bearings. Mechanical systems and signal processing, 15 (2), 287-302, (2001). https://dx.doi.org/10.1006/mssp.2000.1330

7 Yan R. Q., Gao R. X., and Chen X. F. Wavelets for fault diagnosis of rotary machines: a review with applications, Signal Processing, 96, 1-15, (2014). https://dx.doi.org/10.1016/j.sigpro.2013.04.015

8 Huang N. E. and Shen Z. The empirical mode decomposition and the Hilbert spectrum for nonlinear and non-stationary time series analysis. Proceedings of the Royal Society of London A: Mathematical, Physical and Engineering Sciences, 454, 1971, (1998). https://dx.doi.org/10.1098/rspa.1998.0193
9 Dong H. B. and Qi K. Y. Sifting process of EMD and its application in rolling element bearing fault diagnosis. Journal of mechanical science and technology, 23 (8), 2000-2007, (2009). https://dx.doi.org/10.1007/s12206-009-0438-9

10 Wang Y. X., He Z. J., and Zi Y. Y. A comparative study on the local mean decomposition and empirical mode decomposition and their applications to rotating machinery health diagnosis. Journal of Vibration and Acoustics, 132 (2), 021010, (2010). https://dx.doi.org/10.1115/1.4000770

11 Wang Y. H., Yeh C. H., Young H. W. V., Hu K., and Lo M. T. On the computational complexity of the empirical mode decomposition algorithm. Physica A Statistical Mechanics \& Its Applications, 400 (2), 159-167, (2014). https://dx.doi.org/10.1016/j.physa.2014.01.020

$12 \mathrm{Wu}$ Z. H. and Huang N. E. Ensemble empirical mode decomposition: a noise-assisted data analysis method. Advances in adaptive data analysis, 1 (1), 1-41, (2009). https://dx.doi.org/10.1142/S1793536909000047

13 Lei Y. G., He Z. J., and Zi Y. Y. Application of the EEMD method to rotor fault diagnosis of rotating machinery. Mechanical Systems and Signal Processing, 23 (4), 1327-1338, (2009). https://dx.doi.org/10.1016/j.ymssp.2008.11.005

14 Xiang J. and Zhong Y. A fault detection strategy using the enhancement ensemble empirical mode decomposition and random decrement technique. Microelectronics Reliability, 75, 317-326, (2017). https://dx.doi.org/10.1016/j.microrel.2017.03.032

15 Meng L., Xiang J., Wang Y., Jiang Y., and Gao H. A hybrid fault diagnosis method using morphological filter-translation invariant wavelet and improved ensemble empirical mode decomposition. Mechanical Systems \& Signal Processing, 50-51, 101-115, (2015). https://dx.doi.org/10.1016/j.ymssp.2014.06.004

16 Dragomiretskiy K. and Zosso D. Variational mode decomposition. Signal Processing, IEEE Transactions on Signal Process., 63 (3), 531-544, (2014).

17 Wang Y. X. and Markert R. Research on variational mode decomposition and its application in detecting rub-impact fault of the rotor system. Mechanical Systems and Signal Processing, 60, 243-251, (2015). https://dx.doi.org/10.1016/j.ymssp.2015.02.020 
X. Zheng, et al.: APPLICATION OF VARIATIONAL MODE DECOMPOSITION AND PERMUTATION ENTROPY FOR ROLLING BEARING...

Table 4. Classification results based on EMD and PE.

\begin{tabular}{|c|c|c|c|c|c|c|c|}
\hline \multirow{2}{*}{ Fault type } & \multirow{2}{*}{ Test sample } & \multicolumn{3}{|c|}{ Classification results } & \multirow{2}{*}{ Overall } \\
\cline { 3 - 7 } & & Normal status & Inner race fault & Outer race fault & Ball fault & \\
\hline Normal status & 30 & 30 & 0 & 0 & 0 & 100 \\
\hline Inner race fault & 30 & 0 & 30 & 0 & 0 & 100 \\
\hline Outer race fault & 30 & 0 & 5 & 25 & 0 & 83.33 \\
\hline Ball fault & 30 & 0 & 0 & 0 & 30 & 100 \\
\hline
\end{tabular}

Table 5. Classification results based on WTD and PE.

\begin{tabular}{|c|c|c|c|c|c|c|c|}
\hline \multirow{2}{*}{ Fault type } & \multirow{2}{*}{ Test sample } & \multicolumn{4}{|c|}{ Classification results } & \multirow{2}{*}{\begin{tabular}{c} 
Overall \\
\cline { 3 - 7 }
\end{tabular}} \\
\cline { 3 - 7 } Classification rate (\%) & Normal status & Inner race fault & Outer race fault & Ball fault & \\
\hline Normal status & 30 & 30 & 0 & 0 & 0 & 100 \\
\hline Inner race fault & 30 & 0 & 30 & 0 & 0 & 100 \\
\hline Outer race fault & 30 & 0 & 0 & 28 & 26 & 93.33 \\
\hline Ball fault & 30 & 0 & 0 & 4 & 86.67 \\
\hline
\end{tabular}

18 Aneesh C., Kumar S., et al. Performance comparison of variational mode decomposition over empirical wavelet transform for the classification of power quality disturbances using support vector machine. Procedia Computer Science, 46, 372-380, (2015). https://dx.doi.org/10.1016/j.procs.2015.02.033

19 Yang J. Y., Zhang Y. Y., and Zhu Y. S. Intelligent fault diagnosis of rolling element bearing based on SVMs and fractal dimension. Mechanical Systems and Signal Processing, 21 (5), 2012-2024, (2007). https://dx.doi.org/10.1016/j.ymssp.2006.10.005

20 Yan R. Q. and Gao R. X. Approximate entropy as a diagnostic tool for machine health monitoring. Mechanical Systems and Signal Processing, 21 (2), 824-839, (2007). https://dx.doi.org/10.1016/j.ymssp.2006.02.009

21 Zheng J. D., Cheng J. S., and Yang Y. A rolling bearing fault diagnosis approach based on LCD and fuzzy entropy. Mechanism and Machine Theory, 70, 441-453, (2013). https://dx.doi.org/10.1016/j.mechmachtheory.2013.08.014

$22 \mathrm{Wu}$ S. D. and Wu P. H. Bearing fault diagnosis based on multiscale permutation entropy and support vector machine. Entropy, 14 (8), 1343-1356, (2012). https://dx.doi.org/10.3390/e14081343

23 Bandt C. and Pompe B. Permutation entropy: a natural complexity measure for time series. Physical review letters, 88 (7), 174102, (2002). https://dx.doi.org/10.1103/PhysRevLett.88.174102

24 Bruzzo A. A. and Gesierich B. Permutation entropy to detect vigilance changes and preictal states from scalp EEG in epileptic patients. A preliminary study, Neurological sciences, 29 (1), 3-9, (2002). https://dx.doi.org/10.1103/PhysRevLett.88.174102

25 Taherkhani F. and Rahmani M. Permutation entropy and detrend fluctuation analysis for the natural complexity of cardiac heart interbeat signals. Physica A: Statistical Mechanics and its Applications, 392 (14), 3106-3112, (2013). https://dx.doi.org/10.1103/PhysRevLett.88.174102
26 Yan R. Q., Liu Y. B., and Gao R. X. Permutation entropy: a nonlinear statistical measure for status characterization of rotary machines. Mechanical Systems and Signal Processing, 29, 474-484, (2012). https://dx.doi.org/10.1016/j.ymssp.2011.11.022

27 Zhang X. Y., Liang Y. T., and Zhou J. Z. A novel bearing fault diagnosis model integrated permutation entropy. ensemble empirical mode decomposition and optimized SVM, Measurement, 69, 164-179, (2015). https://dx.doi.org/10.1016/j.measurement.2015.03.017

28 Zhao L. Y., Yu W., and Yan R. Q. Gearbox Fault Diagnosis Using Complementary Ensemble Empirical Mode Decomposition and Permutation Entropy. Shock and Vibration, 2016, (2016). https://dx.doi.org/10.1155/2016/3891429

29 Hsu C. W. and Lin C. J. A comparison of methods for multiclass support vector machines. Neural Networks, IEEE Transactions on Signal Processing, 13 (2), 415-425, (2002). https://dx.doi.org/10.1109/72.991427

${ }^{30}$ Hestenes M. R. Multiplier and gradient methods. Journal of optimization theory and applications, 4 (5), 303-320, (1969). https://dx.doi.org/10.1007/BF00927673

31 Smith W. A. and Randall R. B. Rolling element bearing diagnostics using the case western reserve university data: a benchmark study. Mechanical Systems \& Signal Processing, 64-65, 100-131, (2015). https://dx.doi.org/10.1016/j.ymssp.2015.04.021

32 Tang B. Q., Dong S. J., and Song T. Method for eliminating mode mixing of empirical mode decomposition based on the revised blind source separation. Signal Processing, 92 (1), 248-258, (2012). https://dx.doi.org/10.1016/j.sigpro.2011.07.013

33 Cao Y. H. and Tung W. W. Detecting dynamical changes in time series using the permutation entropy. Physical Review E, 70 (4), 046217, (2004). https://dx.doi.org/10.1103/PhysRevE.70.046217 